\title{
Allee Effect in Gause Type Predator-Prey Models: Existence of Multiple Attractors, Limit cycles and Separatrix Curves. A Brief Review
}

\author{
E. González-Olivares*, A. Rojas-Palma \\ Grupo de Ecología Matemática, Instituto de Matemáticas \\ Pontificia Universidad Católica de Valparaíso, Chile.
}

\begin{abstract}
This work deals with the consequences on structural stability of Gause type predator-prey models, when are considered three standard functional responses and the prey growth rate is subject to an Allee effect.

An important consequence of this ecological phenomenon is the existence of a separatrix curve dividing the behavior of trajectories in the phase plane. The origin is an attractor for any set of parameters and the existence of heteroclinic curves can be also shown.

Conditions on the parameter values are established to ensure the existence of a unique positive equilibrium, which can be either an attractor or a repellor surrounded by one or more limit cycles.

The influence of the Allee effect on the number of limit cycles is analyzed and the results are compared with analogous models without this phenomenon, and which main features have been given in various above works. Ecological interpretations of these results are also given.
\end{abstract}

Keywords and phrases: Allee effect, functional response, predator-prey models, limit cycle, bifurcations, separatrix curve.

Mathematics Subject Classification: 92D25, 34C, 58F14, 58F21

\section{Introduction}

In this paper we analyze some Gause type predator-prey models, considering three different prey dependent functional responses and Allee effect on prey growth equation. We describe the consequences of this phenomenon on the behavior of the models, establishing the number of limit cycles that the system can exhibit; we think this number is a good criterion for the classification of models.

The determination of the global bifurcations in these models is critical for understanding their dynamics. Particularly, in the absence of Allee effect, necessary and sufficient conditions for the existence and uniqueness of a limit cycle have been established using the same type of functional responses [40,56].

If the Allee effect is considered, we show the impossibility to establish general properties with respect to the uniqueness or multiplicity of limit cycles for these systems; then, the problem of determining

*Corresponding author. E-mail: ejgonzal@ucv.cl 
conditions guaranteing the uniqueness or multiplicity of limit cycles surrounding a positive equilibrium remains open [15].

Gause type models considering Allee effect on the prey growth equation are sensitive to the mathematical form used for the predator functional response. Also, different dynamical behaviors can exist, such as: stable coexistence of both species, persistent oscillations, predator extinction or extinction of both species [57].

This paper is organized as follows: In the subsequent subsection, the problem of determining the number of limit cycles surrounding a positive equilibrium point in predation models is described; later, a brief description of the Allee effect and the types of functional response is given. The main properties of models in the absence of the Allee effect are also presented.

In Section 2, models topologically equivalent to the Gause type predator-prey model are obtained; in Section 3, the main properties of these models are proved. Finally, some ecological consequences of the mathematical results and a table summarizing the number of limit cycles of the models studied are given in Section 4.

\subsection{Continuous-time Gause type predator-prey models}

The models here studied were named after the Russian biologist, Georgii Frantsevich Gause (b. 1910, d. 1986), who in 1932 proposed the competitive exclusion principle, based on experimental work done with mixed cultures of yeast species and Paramecium cultures [28]. This work culminated in 1934 in the important publication entitled The Struggle for Existence [26].

A lot of work has been done on the existence and uniqueness of limit cycles in a predator-prey interaction modeled by bidimensional autonomous differential equations systems. One well-known version of such systems, the so-called Gause type models [22], has the following general form:

$$
G:\left\{\begin{array}{l}
\frac{d x}{d t}=x g(x)-h(x) y \\
\frac{d y}{d t}=(\psi(x)-c) y
\end{array}\right.
$$

where $x(t)$ and $y(t)$ indicate the prey and predator population sizes at time $t>0$, respectively, and $c>0$ is the natural per capita death rate of predators. The functions $f(x)=x g(x), h(x)$ and $\psi(x)$ have appropriate properties $[48,77]$ representing the prey growth rate, the functional response or trophic function of the predator [40] and the numerical response, respectively.

Global analysis of model (0) has been studied in several articles by considering the case where $\psi(x)=$ $p h(x)$, with $p>0$ being the conversion efficiency rate of prey to predators, that is, the system satisfies the conversion rule [56]. For $\psi(x) \neq h(x)$, there are little known results on the existence and uniqueness of limit cycles of (0) for some rather general families of predator responses [56]. They can be used to explain many real world oscillatory phenomena in nature [40,77], such as snowshoe hares and lynx in northern Canada, house sparrows and sparrow hawks in Europe, muskrats and mink in central North America, bighorn sheep and wolves in Alaska [43].

The problem of determining conditions, which guarantee the uniqueness of a limit cycle or the global stability of the unique positive equilibrium in predator-prey systems, has been extensively studied over the last three decades [40], starting with the work by Cheng [10], who first proved the uniqueness of a limit cycle for a specific predator-prey model with a Holling type-II functional response, using the symmetry of the prey isocline [77].

Although it is possible to build models with many limit cycles [43,77], we will only analyze those presented in the ecological literature, primarily because we are motivated by C. S. Coleman [15], who suggested: "Find a predator prey or other interacting system in nature, or construct one in the laboratory, with at least two ecologically stable cycles". Mathematically, a system representing this situation must have at least, three limit cycles. In this context, ecologically stable means a natural cycle persevering over a long period of time which must be somewhat insensitive to the inevitable shocks and disturbances of the real world [15]. 
The latter is related to the unsolved problem proposed by David Hilbert in 1900 (Hilbert's 16th Problem), and refers to finding the maximum number of limit cycles of a bidimensional polynomial differential equation system, which degree must be equal to $p \in \mathbb{N}[24]$.

\subsection{Models for the Allee effect}

Different equations have been suggested for the prey growth rate for single species, which have been classified as compensatory or depensatory equations [13]. The most common among the first, is the well-known logistic model, but other compensatory equations have been used in the ecological literature [5] as the Gompertz [53] or Pella-Tomlinson equation [13]. In the book by C. W. Clark, it is said that compensatory equation has a behavior similar to the logistic growth equation.

Depensatory equations are used to model the Allee effect in situations occurring at low population densities, where the per-individual growth rate is an increasing function of population abundance [65]. This effect is named after Warder Clyde Allee, an American zoologist and ecologist (b.1885 d.1955) [19], who was the first to study this phenomenon.

The Allee effect is defined as: a positive relationship between a component of individual fitness and the number or density of conspecifics [66]. Thus, any mechanism affecting some measurable component of individual fitness should be included within the definition of Allee effects [65].

This phenomenon has received different names, such as: depensation in Fisheries Sciences, or negative competition effect, inverse density dependence or positive density dependence in Population Dynamics [51]. Populations can exhibit Allee effect due to a wide range of biological phenomena, such as reduced antipredator vigilance, social thermoregulation, genetic drift, mating difficulty, and deficient feeding at low densities; however, several other causes may lead to this phenomenon (see Table 1 in [6] or Table 2.1 in $[19])$.

The dynamic consequences of the Allee effect have been studied within different biological contexts, such as metapopulation dynamics [46], biological invasions [73], infectious diseases [42], or driven by predation [25], etc.

Recent ecological research suggests that two or more Allee effects can lead to mechanisms simultaneously acting on a single population (see Table 2 in [6]); the combined influence of some of these phenomena is known as multiple (double) Allee effect $[2,6]$.

The Allee effect can be divided into two main types called strong Allee effect [71,73] or critical depensation $[13,14,51]$ and weak Allee effect $[66,73]$ or noncritical depensation $[13,14,51]$.

The most common mathematical form for the Allee effect $[5,47]$ is described by the non-linear differential equation

$$
\frac{d x}{d t}=r\left(1-\frac{x}{K}\right)(x-m) x
$$

where $-K \leq m<K$. since the Allee effect is a phenomenon that occurs at low population sizes. The parameter $m>0$, is called the minimum viable population size; $r, K>0$ are the potential biotic [12] or intrinsic rate of population growth $[53,70]$ and the environmental carrying capacity, respectively.

Equation (1) can be obtained by assuming a density-dependent per capita mortality rate (analogously to logistic growth) and a quadratic per capita fertility rate [42]. This equation expresses a weak Allee effect when $m \leq 0$, but here we will consider only the collapse of the singularities $m$ and 0 and $m>0$.

The marked differences between standard logistic growth and population growth incorporating Allee effects are illustrated below in Figure 1, for diverse values of $m$ in equation (1). In the vertical axis, we have plotted the per capita growth of species as a function of population size, comparing the negative density dependence (associated to logistic growth rate, represented by the unbroken line) with the population growth incorporating Allee effects. The dotted line shows growth with this phenomenon that evidently produces a positive slope over a large range of population sizes. The strong Allee effect implies the existence of a threshold population level $m[5,9,17]$, below which the population becomes extinct. This requires the population growth $d x / d t$ to be negative at $x<m$, and positive at $x>m$, where $x=x(t)$ indicates the population size. 


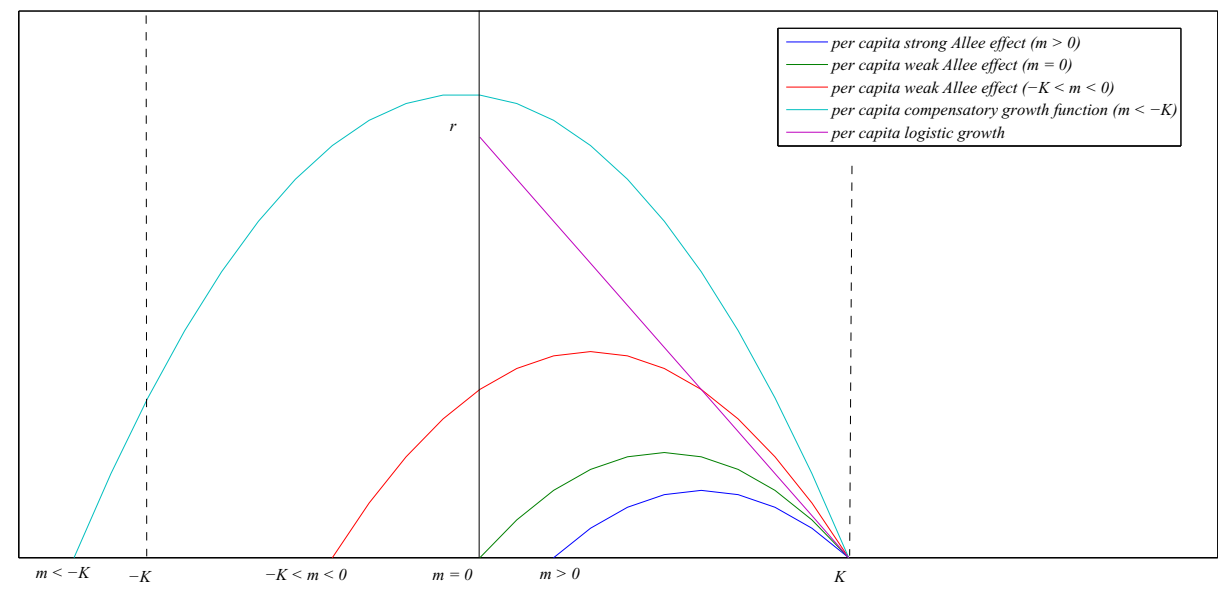

FiguRE 1. Relationships between the per capita population growth rate $f(x)=\frac{1}{x} \frac{d x}{d t}$ andthe population size $x$ (density, biomass or number) are shown, for negative density dependence (straight line), strong, weak and special weak Allee effects $(m>0, m<$ $0, m=0)$, are shown for equation (1). A weak Allee effect occurs if the per capita population growth rate increases with increasing population size and the per capita population growth rate is positive for low population sizes. For $m<-K$, a compensatory curve growth is obtained and the per capita population growth rate $f(x)$ is negative for all $x>0$; this case is not an Allee effect.

Many continuous time equations have been used to model the Allee effect $[8,17,65,78]$, but it is possible to prove the topological equivalence between these different mathematical expressions modelling this phenomenon [31]. This implies the differential equations have the same unidimensional dynamical properties, i.e., solutions have the same qualitative behavior for $x \geq 0$.

Nevertheless, when forms distinct to equation (1) are incorporated on predator-prey models, it can produce a change in the number of limit cycles surrounding a positive equilibrium point and generated by Hopf bifurcation $[21,33,78]$. So, we consider that this issue is a good criterion to classify these models.

\subsection{The functional response}

Function $h(x)$ in equation (0), named the functional response of predators or consumption rate function, the rate at which an individual predator kills prey [70], refers to the change in the density of prey consumed per unit time per predator as the prey population size changes.

As prey population size increases, the response of the predator $h(x)$ may change in various ways (linearity, at a decelerating rate, sigmoidally or initially increasing to a maximum rate and then decrease and saturate at a minimum rate). The ecological literature suggest three basic types of functional response, which involve:

i) The Holling type I functional response is a piecewise-continuous function, also called Blackman's equation [62], which is a monotonically increasing function that tends towards and reaches $c>0$ as the prey size increases [62]. It generally suggests that only filter feeders satisfy the handling and satiation conditions that lead to this type of function [62].

However, the linear form is most commonly used, as in the well-known Lotka-Volterra model [53], being similar to the harvest function $h(x)=q x E$, established by the Schaeffer's Hypothesis in Fisheries Sciences [13].

ii) The Holling type II, concave downward and approaching a horizontal positive asymptote [22], is widely used in the ecological literature, particularly, the form known as the hyperbolic functional response 
[70]. However, considering a monotonic non-differentiable functional response, the behavior of predatorprey model strongly differs from those assuming the hyperbolic functional response [72].

iii) The Holling type III or S-shaped [22], biologically explains the fact that at small densities of prey populations, the effect of predation is low [52]; but, if the population size increases, then the predation is more intensive. This phenomenon appears in real world interactions [55] and the predator is said to be generalist [64], since if the prey population size is low, then it will seek other food alternatives [52].

In this work, the following rational functional responses will be considered for system (0):

i) linear response $h(x)=q x$, where $q$ is the per capita attack rate of predators,

ii) hyperbolic response $h(x)=\frac{q x}{x+a}$, where $q$ is the maximum killing rate and $a$ is the half-saturation $[30,54]$ or Michaelis-Menten constant [39], i.e., prey population size at which the killing rate is half of the maximum [70],

iii) sigmoid response $h(x)=\frac{q x^{2}}{x^{2}+a^{2}}$, where $q$ is the maximal relative biomass growth rate of the predator [40] and $a$ is the half-saturation constant for the predator [68] or the prey abundance where predator satiation begins [64].

We note that

a) the measure unit of parameter $q$ in the linear functional response is $\left[t^{-1}\right]$, whereas in hyperbolic and sigmoid functional responses it is [prey measure unit] $\times\left[t^{-1}\right]$,

b) in this Holling type II and III functional response, the measure unit of $a$ is the same that $x$.

Moreover, in the hyperbolic and sigmoid functional responses, the parameter $a$ measures the abruptness of the functional response [27]. If $a \rightarrow 0$, the curve grows quickly; if $a \rightarrow K$, the curve increases slowler, i.e. a larger quantity of prey is necessary to attain $\frac{q}{2}$, which can also occurs when $a \geq K$. So, in the following, we assume $a<K$.

Many other mathematical formualtions can be proposed for each basic type of fucntional response such as the Rosenzweig functional response described by $h(x)=q x^{\alpha}[53,59]$, which is non-differentiable at $x=0$ or the Ivlev functional response [53], a trascendent function given by $h(x)=q\left(1-e^{-a x}\right)$, in which the consumption rate increases at a decreasing rate with prey density until it becomes constant at satiation [56], i.e. it is also monotonically increasing and asymptotics to a fixed value.

Also, different types of functional response such as the Holling type IV [69], ratio-dependent [7,63], Beddington-DeAngelis [44], or Hassel-Varley [45] have been included on the formulation of predatorprey models However, some of these forms can produce strong changes in the dynamics of the model $[7,44,45,63]$, and particularly, in the quantity of limit cycles surrounding a positive equilibrium point in predator-prey models $[1,21]$.

\subsection{Models with logistic prey growth}

Properties of system (0) considering the logistic growth function for the prey and increasing functional responses, are well-known. The equilibrium points $(0,0),(K, 0)$ always exist. The point $\left(x_{e}, y_{e}\right)$ lies in the first quadrant for certain constraints on the parameter space and in this case $(0,0)$ and $(K, 0)$ are hyperbolic saddle points.

In the Lotka-Volterra model [53], the global stability the positive equilibrium point $\left(\frac{c}{p}, y_{e}\right)$ is established by means of the following Lyapunov function proposed by Goh [29]

$$
V(x, y)=c_{1}\left(x-x_{e}-x_{e} \ln \frac{x}{x_{e}}\right)+c_{2}\left(y-y_{e}-y_{e} \ln \frac{y}{y_{e}}\right)
$$

In the Rosenzweig-MacArthur (considering the hyperbolic functional response) and in the Yodzis model [70] (incorporating the sigmoid functional response), when the point $\left(x_{e}, y_{e}\right)$ lies in the first quadrant, it can be an attractor or a repellor surrounded by a unique limit cycle $[39,67]$

For the Rosenzweig-MacArthur and Yodzis models [70], local stability of $\left(x_{e}, y_{e}\right)$ implies global asymptotically stability of this point. Moreover, in $[39,56]$ it is shown that the existence of a limit cycle is equivalent to the unstability of the unique positive equilibrium point of the model. There assuming the logistic growth function for the prey and a general functional response in which the consumption rate increases at a decreasing rate with prey population size until it becomes constant at satiation (as 
the Holling Type II and III functional response) [56]. Table 1 summarizes the nature of the equilibrium points on the Rosenzweig-MacArthur model.

Table 1. Equilibrium points and their nature in the Rosenzweig-MacArthur model, where $c>0$ is the natural per capita death rate of predators, $p>0$ is the conversion efficiency rate of prey to predators and $K>0$ environmental carrying capacity.

\begin{tabular}{|c|c|c|c|}
\hline$\frac{\text { Equilibrium point } \rightarrow}{\text { Parameter constraints } \downarrow}$ & $(0,0)$ & $(K, 0)$ & $\left(\frac{a c}{K(p-c)},\left(1-\frac{a c}{K(p-c)}\right)\left(\frac{a c}{K(p-c)}+\frac{a}{K}\right)\right)$ \\
\hline$\frac{a c}{K(p-c)}>1$ & saddle & global attractor & out of first quadrant \\
\hline$\frac{a c}{K(p-c)}<1, a>K-2 \frac{a c}{p-c}$ & saddle & saddle & global attractor \\
\hline$\frac{a c}{K(p-c)}<1, a<K-2 \frac{a c}{p-c}$ & saddle & saddle & repellor with a limit cycle \\
\hline
\end{tabular}

Moreover, in [41] a sufficient and necessary condition for the existence of limit cycles is given, when the derivative of the functional response is positive, decreasing, and concave upward.

However, these assertions are not true in general. Choosing a monotonically increasing but nondifferentiable functional response for $x=0$, it is possible to demonstrate the existence of a separatrix curve dividing the phase plane and two limit cycles surrounding a unique positive equilibrium point [72].

\section{The Models}

Now, we focus on the dynamics of Gause-type predator-prey model, considering the Allee effect on prey modeled by equation (1) and some of the functional responses outlined above. This models are described by the following autonomous Kolmogorov type bidimensional differential equation system [22, 29]:

$$
X_{\mu}:\left\{\begin{array}{l}
\frac{d x}{d t}=r\left(1-\frac{x}{K}\right)(x-m) x-h(x) y \\
\frac{d y}{d t}=(p h(x)-c) y
\end{array}\right.
$$

where $x=x(t)$ and $y=y(t)$ indicate the prey and predator population size, respectively, with $t>0$ (measured as the number of individuals, density or biomass), and $h(x)$ is the functional response.

In system (2), all parameters are positive and by ecological reason $a<K$ and $m<K$, i.e., $\mu=$ $(r, K, q, c, p, a, m) \in \mathbb{R}_{+}^{5} \times\left[0, K{ }^{2}\right.$. System (2) is defined in the first quadrant:

$$
\Omega=\left\{(x, y) \in \mathbb{R}^{2} / x \geq 0, y \geq 0\right\}=\mathbb{R}_{0}^{+} \times \mathbb{R}_{0}^{+} .
$$

The equilibrium points of system (2), or singularities of the vector field $X_{\mu}$, are $O=(0,0), P_{K}=(K, 0)$, $P_{m}=(m, 0)$ and $P_{e}=\left(x_{e}, y_{e}\right)$, which are unique when using the linear functional response, the Holling type II and III functional response. Assuming a non-monotonic functional response in system (2), one, two or none positive equilibrium points can exist [38].

\subsection{Topologically equivalent systems}

In order to simplify the calculations, we make a change of variables and a time rescaling given by the function $\varphi: \tilde{\Omega} \times \mathbb{R} \longrightarrow \Omega \times \mathbb{R}$

with

and

$$
\tilde{\Omega}=\left\{(u, v) \in \mathbb{R}^{2} / u \geq 0, v \geq 0\right\}=\mathbb{R}_{0}^{+} \times \mathbb{R}_{0}^{+}
$$

$$
\varphi(u, v, \tau)=\left(K u, \frac{L}{q} v, \frac{1}{r K} h_{1}(x) \tau\right)=(x, y, t) .
$$

where $L=r K$ [34] or $r K^{2}$ [35], and $h_{1}(x)=1$ [37] or $u+\frac{a}{K}$ [34], or $u^{2}+\frac{a^{2}}{K^{2}}$ [35, 36], according to the functional response employed. Thus, $\operatorname{det} D \varphi(u, v, \tau)>0$; in particular, for the model considering sigmoid functional response, we have that $\operatorname{det} D \varphi(u, v, \tau)=\frac{1}{q}\left(a^{2}+K^{2} u^{2}\right)$. 
Then, $\varphi$ is a diffeomorphism [11] preserving the time orientation; hence, we obtain a qualitatively (topologically) equivalent vector field $Y_{\nu}=\varphi \circ X_{\mu}$, which has the form $Y_{\nu}=P(u, v) \frac{\partial}{\partial u}+Q(u, v) \frac{\partial}{\partial v}$ [20]. The associated polynomial differential equation systems are also of Kolmogorov type [22] and we have:

i) For the linear functional response

$$
L_{\nu}:\left\{\begin{array}{l}
\frac{d u}{d \tau}=((1-u)(u-M)-v) u \\
\frac{d v}{d \tau}=B(u-C) v
\end{array}\right.
$$

where $\nu=(B, C, M) \in \mathbb{R}_{+}^{2} \times[0,1[$, with

$$
B=\frac{p}{r}, C=\frac{c}{p K} \text { and } M=\frac{m}{K} .
$$

ii) For the hyperbolic functional response

$$
H_{\eta}:\left\{\begin{array}{l}
\frac{d u}{d \tau}=((1-u)(u-M)(A+u)-v) u \\
\frac{d v}{d \tau}=B(u-C) v
\end{array}\right.
$$

where $\eta=(A, B, C, M) \in] 0,1] \times \mathbb{R}_{+}^{2} \times[0,1[$, with

$$
A=\frac{a}{K}, C=\frac{a c}{K(p-c)}, B=\frac{p-c}{r K} \text { and } M=\frac{m}{K} \text {, and } p-c>0 .
$$

iii) For the sigmoid functional response

$$
S_{\eta}:\left\{\begin{array}{l}
\frac{d u}{d \tau}=\left((1-u)(u-M)\left(A+u^{2}\right)-u v\right) u \\
\frac{d v}{d \tau}=B\left(u^{2}-C\right) v
\end{array}\right.
$$

where $\eta=(A, B, C, M) \in] 0,1\left[\times \mathbb{R}_{+}^{2} \times[0,1[\right.$, with

$$
A=\frac{a^{2}}{K^{2}}, B=\frac{p-c}{r K}, C=\frac{a c}{K^{2}(p-c)} \text { for } p-c>0, \text { and } M=\frac{m}{K} .
$$

\section{Main Results}

For the systems of differential equations (3), (4) and (5) we have

Lemma 3.1. Existence of an invariant region

The set $\tilde{\Gamma}=\{(u, v) \in \tilde{\Omega} / 0 \leq u \leq 1, v \geq 0\}$ is an invariant region.

Proof. In system (3), if $u=0$, then $\frac{d u}{d \tau}=0$ and $\frac{d v}{d \tau}=-B C v<0$ and the trajectories remain over the $v$-axis.

If $v=0$, then $\frac{d v}{d \tau}=0$ and

$\frac{d u}{d \tau}=(1-u)(u-M) u>0$ if $u>M$.

If $u=1$, then $\frac{d u}{d \tau}=-v u<0$ and $\frac{d v}{d \tau}=B(1-C) v$, which can be positive or negative, but trajectories point towards the interior of $\tilde{\Gamma}$.

For the other systems, the proof is similar.

\section{Lemma 3.2. Boundedness of the solutions}

The solutions are bounded.

Proof. In order to prove the boundedness of solutions, the Poincaré compactification [11] is used to study the behavior of point $(0, \infty)$. Through the change of variables $X=\frac{u}{v}$ and $Y=\frac{1}{v}$, we have that $\frac{d X}{d \tau}=\frac{1}{v^{2}}\left(v \frac{d u}{d \tau}-u \frac{d v}{d \tau}\right)$ and $\frac{d u}{d \tau}=-\frac{1}{v^{2}} \frac{d v}{d \tau}$. After simplifications, a new system $\breve{Z}$ is obtained, where the nature of the point $(0,0)$ of $\breve{Z}$ determines the nature of $(0, \infty)$.

Using the blowing up method [11,20], it is shown that $(0,0)$ is a non-hyperbolic saddle point and, as a consequence, the point $(0, \infty)$ is a saddle point.

The following results were obtained for system of differential equations (3), (4) and (5). 
Lemma 3.3. Nature of equilibrium points on axes

2.1) The equilibrium point $Q_{1}=(1,0)$ is

2.1.1) a hyperbolic attractor, if and only if, $C>1$.

2.1.2) a hyperbolic saddle point, if and only if, $0<M<C<1$.

2.1.3) a non-hyperbolic attractor, if and only if, $C=1$.

2.2) The point $Q_{M}=(M, 0)$ is

2.2.1) a hyperbolic saddle point, if and only if, $M<C$.

2.2.2) a hyperbolic repellor, if and only if, $C<M$.

2.2.3) a non-hyperbolic repellor, if and only if, $M=C$.

2.3) The equilibrium point $O=(0,0)$ is a hyperbolic attractor for any set of parameter values

Proof. The results are directly obtained from the evaluation of the Jacobian matrix.

Remark 3.4. Let $W^{s}(M, 0)$ and $W^{u}(1,0)$ be the stable and unstable manifold of the equilibrium point $(M, 0)$ and $(1,0)$, respectively. Then, there exists a separatrix curve $\Sigma$ in $\bar{\Gamma}$ determined by $W^{s}(M, 0)$, which divides the behavior of the trajectories (Fig. 2a).

Let $u_{0}$ be such that $0<M \leq u_{0} \leq 1$ and the points $\left(u_{0}, v^{s}\right) \in W^{s}(M, 0)$ and $\left(u_{0}, v^{u}\right) \in W^{u}(1,0)$ are in the intersection of the straight line $u=u_{0}, W^{s}(M, 0)$ and $W^{u}(1,0)$, respectively.

If $v^{s}>v^{u}$, an invariant subregion $\Lambda$ exists, determined by the stable manifold $W^{s}(M, 0)$ of $(M, 0)$, the straight line $u=1$ and the $u$-axis, denoted by $\Lambda$, i.e.

$$
\Lambda=\left\{(u, v) \in \tilde{\Gamma} / M \leq u \leq 1,0 \leq v \leq v^{s}\right\}
$$

is a compact region and the Poincaré-Bendixson Theorem applies (see Figure 2(a)).

Then, the main consequence of the strong Allee effect is a separatrix curve [4] appearing in the phase plane determined by the stable manifold $W^{s}(M, 0)$ of the saddle point $(M, 0)$, which divides the region $\tilde{\Gamma}$ into two subregions, one of them denoted by $\Lambda$.

Therefore, trajectories are highly sensitive to initial conditions. This implies that there is a high possibility that both populations go to extinction and in the situation described by Fig. 2b, depending on the ratio between population sizes, both populations could either reach extinction or long-term coexistence $[33,34]$.
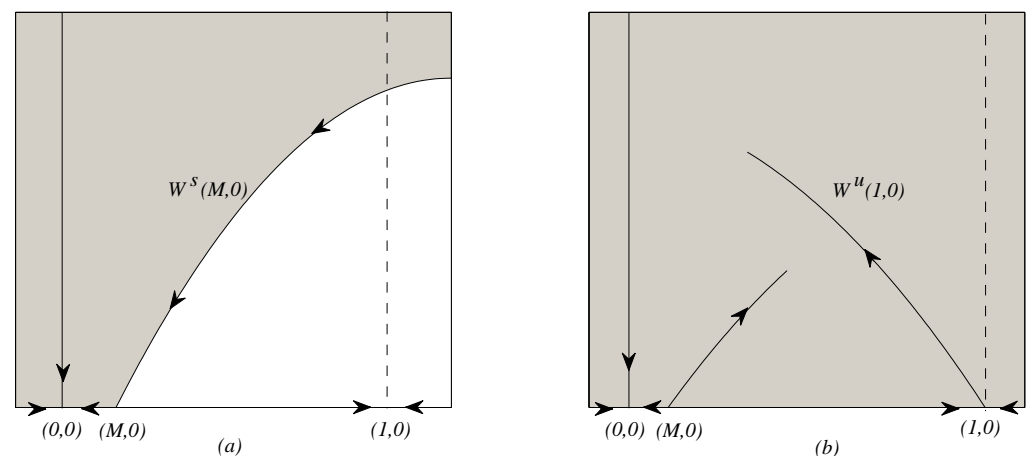

Figure 2. The trajectories with initial conditions in the dark zones have the point $(0,0)$ as their $\omega$-limit, for $E>1$ or $0<E<M$.

We note that for the differential equation systems (3), (4) and (5):

a) If $C>1$, then there are three equilibrium points $O, Q_{1}$ and $Q_{M}$ in the invariant region $\tilde{\Gamma}$. The equilibrium point $O$ and $Q_{1}$ are local attractors, while $Q_{M}$ is a saddle point. The point $Q_{e}$ lies outside 
the first quadrant and the trajectories with initial conditions inside $\Lambda$ have the point $Q_{1}$ as $\omega-$ limit, whereas the orbits starting out of $\Lambda$ (over the manifold $W^{s}(M, 0)$ ) have the point $(0,0)$ as their $\omega$-limit (Fig. 2a).

b) If $C=1$, then $Q_{e}$ collapses with $Q_{1}$ and a non-hyperbolic local attractor is obtained; the point $O$ is then a local attractor and $Q_{M}$ a saddle point.

c) If $M<C<1$, then there are four equilibrium points $O, Q_{1}, Q_{M}$ and $Q_{e}$ in the invariant region $\bar{\Gamma}=\{(u, v) \in \tilde{\Gamma} / M<u<1, v \geq 0\}$. The point $O$ is a local attractor and $Q_{1}$ a saddle point; the nature of $Q_{M}$ and $Q_{e}$ must be determined.

d) If $C=M$, then $Q_{e}$ collapses with $Q_{M}$, which is a non-hyperbolic repellor, and $O$ is a global attractor.

e) If $C<M$, then there are three equilibrium points in the invariant region: $Q_{1}$ is a saddle point, $Q_{M}$ is a repellor, and $O$ is a global attractor for the trajectories in $\tilde{\Gamma}$ (Fig. 2b).

In what follows, we assume in the differential equations systems (3), (4) and (5) $0<M<C<1$. Hence the existence of a unique equilibrium point $Q_{e}$ in the interior of the first quadrant is guaranteed, more precisely in the subregion $\bar{\Gamma}$. Moreover, the equilibrium point $O$ is a local attractor and the singularities $Q_{1}$ and $Q_{M}$ are saddle points.

As $\operatorname{det} D Y_{\nu}(C, L)>0$, the nature of the equilibrium point $Q_{e}$ is dependent on the sign of the trace of the Jacobian matrix evaluated at this point; $Q_{e}$ can either be an attractor or a repellor surrounded by at least one limit cycle (Poincaré -Bendixon theorem), or else, it is a weak focus [11] in the subregion $\Lambda$.

Whether $Q_{e}$ is a node or a focus depends on the quantity

$$
P=\left(\operatorname{tr} D Y_{\nu}(C, L)\right)^{2}-4 \operatorname{det} D Y_{\nu}(C, L) \text {. }
$$

In Figure 3, when $(C, L) \in \bar{\Gamma}$, the different positions of $W^{s}(M, 0)$ and $W^{u}(1,0)$, the stable and unstable manifolds of $Q_{M}$ and $Q_{1}$, respectively, are shown.
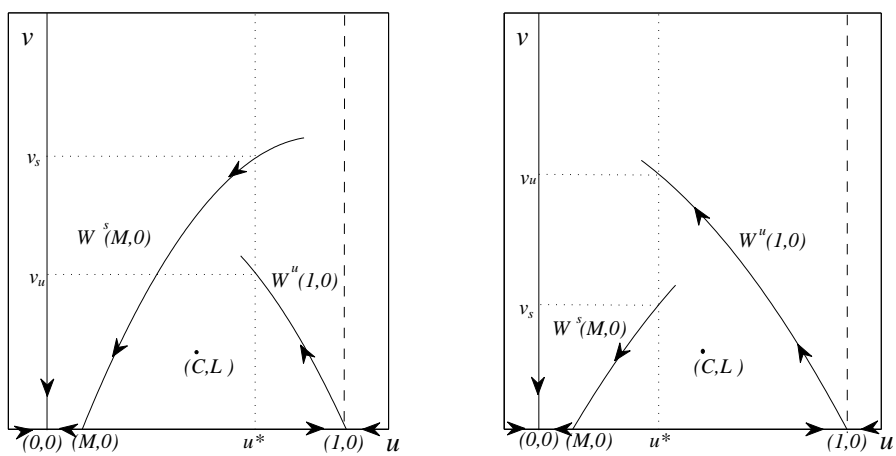

Figure 3. Different positions of the stable manifold $W^{s}$ of saddle point $(M, 0)$ and the unstable manifold $W^{u}$ of saddle point $(1,0)$, when $0<M<E<1$, in systems (3)-(5). In left poster the stable manifold $W^{s}$ of $(M, 0)$ is above the unstable manifold $W^{u}$ of $(1,0)$, meanwhile in the right poster the stable manifold $W^{s}$ of $(M, 0)$ is below the unstable manifold $W^{u}$ of $(1,0)$. By continuity of systems with respect to the parameter values, there exists a condition (a surface) in the parameter space for which both manifolds intersect.

Lemma 3.5. Let $W^{s}(M, 0)$ and $W^{u}(1,0)$ be the stable and unstable manifold of $Q_{M}$ and $Q_{1}$, respectively; then, a subset of parameter values exists, for which $W^{s}(M, 0)=W^{u}(1,0)$, givie rise to a heteroclinic curve $\gamma_{1 M}$, joining the saddle points $Q_{1}$ and $Q_{M}$. 
Proof. It is clear that the $\alpha$-limit of $W^{s}(M, 0)$ and the $\omega$-limit of $W^{u}(1,0)$ are not at infinity in the direction of the $v$-axis, since the solutions are bounded.

Neither the $\omega$-limit of $W^{u}(1,0)$ is over the $u$-axis, except the point $(M, 0)$, nor can it be the same point $(1,0)$, since there is no homoclinic curve.

Let $u^{*}$ be such that $0<M \leq u \leq 1$; then, there are points $\left(u^{*}, v^{s}\right) \in W^{s}(M, 0)$ and $\left(u^{*}, v^{u}\right) \in$ $W^{u}(1,0)$, with $v^{s}$ and $v^{u}$ dependent on the parameters, i.e. $v^{s}=F(A, B, C, M)$ and $v^{u}=G(A, B, C$, $M)$.

If $0<M<u^{*}<<1$, then $v^{s}<v^{u}$ and the stable manifold $W^{s}(M ; 0)$ is below the unstable manifold $W^{u}(1 ; 0)$.

If $0<M<<u^{*}<1$, then $v^{s}>v^{u}$ and the stable manifold $W^{s}(M ; 0)$ is above the unstable manifold $W^{u}(1 ; 0)$ (see Fig 3$)$

Since systems (3), (4) and (5) are continuous with respect to the parameter values, the stable manifold $W^{s}(M, 0)$ intersects the unstable manifold $W^{u}(1,0)$. Moreover, by uniqueness of solutions of the systems, this intersection must occur along a trajectory joining the saddle points $(1 ; 0)$ and $(M ; 0)$.

Hence, there exists $\left(u^{*}, v^{*}\right) \in \bar{\Gamma}$, such that $v^{s}=v^{u}=v^{*}$ and the equation $F(A, B, C, M)=G(A, B, C$, $M)$ defines a surface in the parameter space $\Delta$, for which the heteroclinic curve $\gamma_{1 M}$ exists.

In the bounded invariant subregion $\tilde{\Gamma} \subset \bar{\Gamma}$ the frontier is determined by the subinterval $[M, 1]$ and the heteroclinic curve.

Remark 3.6. We note the heteroclinic curve $\gamma_{1 M} \subset W^{s}(M, 0) \cap W^{u}(1,0)$ and it lies entirely on a segment of the $u$-axis and exists for any parameter value such that $0<M<C<1$.

It follows that a heteroclinic cycle (loop) $\gamma_{h}$ exists for certain parameter values on the same submanifold. More precisely, $\gamma_{h}=(1,0) \cup \gamma_{1 M} \cup(M, 0) \cup \gamma_{M 1}$.

In Figure 4, the existence of the heteroclinic curve in system (3) is shown [71].

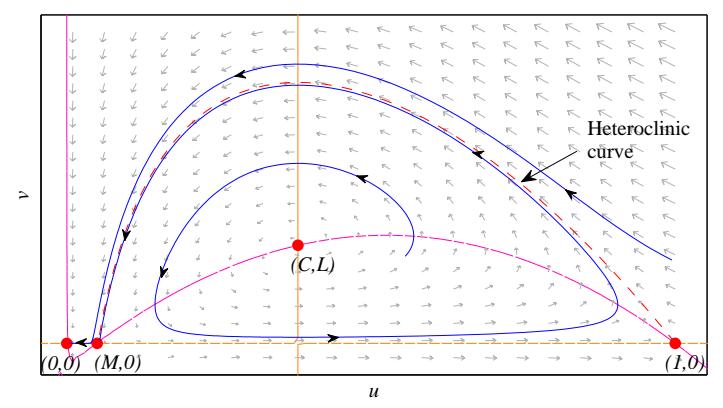

Figure 4. Heteroclinic curve determined by the equilibrium points $(M, 0)$ and $(1,0)$ with $M=0.05, C=0.38$ and $B=1$, in system (3).

\subsection{Nature of the unique positive equilibrium point}

In the systems of differential equations (3), (4) and (5) we have:

Theorem 3.7. Assuming that $v^{s}<v^{u}$ and $M<C \ll 1$, the equilibrium point $Q_{e}$ is a repelling node and the equilibrium point $(0,0)$ is a global attractor. Thus, there exists a new heteroclinic curve linking the points $Q_{e}$ and $(0,0)$. 
Proof. If $v^{s}<v^{u}$, then the stable manifold $W^{s}(M, 0)$ lies under the unstable manifold $W^{u}(1,0)$ and the point $(C, L)$ is a repellor node. Then, by the Existence and Uniqueness Theorem, the trajectories with initial conditions near the point $(C, L)$, cannot intersect with $W^{u}(1,0)$. Since $(M, 0)$ and $(1,0)$ are saddle points, the trajectories have the origin $(0,0)$ as their $\omega$-limit, and this equilibrium point is then globally asymptotically stable.

The situation described in the above theorem is shown in figure 5 for system (4).

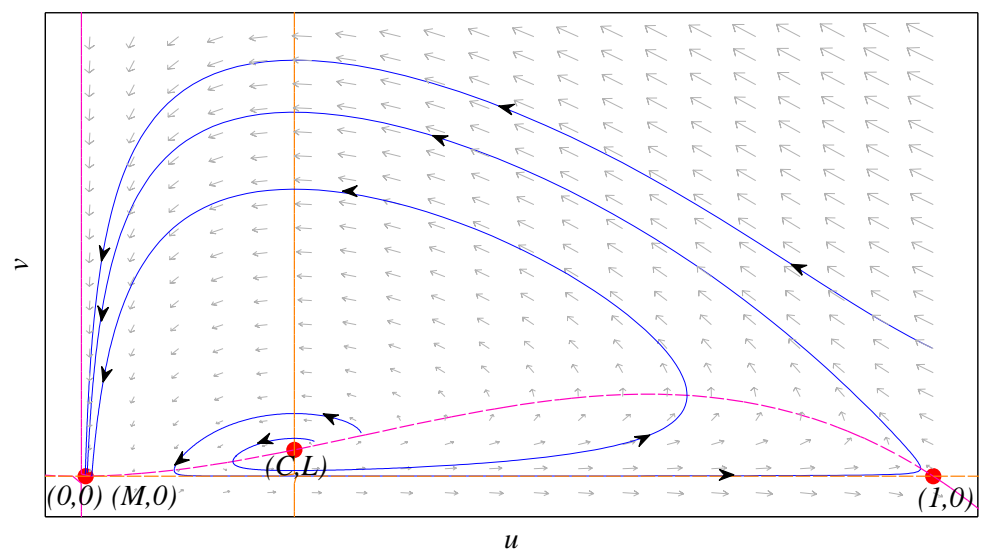

Figure 5. Extinction of two species in system (4). For $A=0.01 ; B=1, C=0.25$ and $M=0.005$; the singularity $(0,0)$ is a global attractor equilibrium point; $(C, L)$ is a repellor, the limit cycle has disappeared; $(1,0)$ and $(M, 0)$ are saddle points and the stable manifold $W^{s}$ of $(M, 0)$ is below the unstable manifold $W^{u}$ of $(1,0)$ as is indicated by the right poster of Figure 3, but it cannot be depicted explicitly in this simulation, due to limitations of the program used.

Now, we assume that $v^{s} \geq v^{u}$; then, we have the following properties for the unique equilibrium point $(C, L)$ in systems of differential equations (3), (4) and (5).

Theorem 3.8. Nature of $Q_{e}=(C, L)$

In system (3)

3.1) if $C>\frac{1+M}{2}$, then $\operatorname{tr} D Y_{\nu}(C, L)<0$ and the equilibrium point $(C, L)$ is a hyperbolic local attractor.

3.2) if $C<\frac{1+M}{2}$, then the equilibrium point $(C, L)$ is a hyperbolic repellor.

3.2.1) If $B>\frac{1}{4} C \frac{(-2 C+M+1)^{2}}{(1-C)(C-M)}$, the point $(C, L)$ is a repellor focus surrounded by a limit cycle.

3.2.2) If $B<\frac{1}{4} C \frac{(-2 C+M+1)^{2}}{(1-C)(C-M)}$, the point $(C, L)$ is a repellor node, and all the trajectories of the system have the equilibrium point $(0,0)$ as their $\omega$-limit, $i$. e., the equilibrium $(0,0)$ is globally asymptotically stable.

In system (4)

4.1) If $A>\frac{-3 C^{2}+2 C M+2 C-M}{2 C-M-1}$, then the trace is negative and the equilibrium $(C, L)$ is a hyperbolic local attractor. 
4.2) If $A<\frac{-3 C^{2}+2 C M+2 C-M}{2 C-M-1}$, then the trace is positive and the equilibrium $(C, L)$ is a hyperbolic repellor.

4.2.1) If $B>\frac{\left(-3 C^{2}-2 C A+2 C+A\right)^{2}}{4(1-C)(A+C)}$, then $(C, L)$ is an unstable focus surrounded by a stable limit cycle.

4.2.2) If $B<\frac{\left(-3 C^{2}-2 C A+2 C+A\right)^{2}}{4(1-C)(A+C)}$, then $(C, L)$ is an unstable node and the limit cycle disappears. In this last case, the singularity $(0,0)$ is globally asymptotically stable.

In system (5) the singularity $(C, L)$ is

5.1) a hyperbolic local attractor, if and only if,

$$
-3 C^{4}+2(1+M) C^{3}-(M+A) C^{2}+A M<0,
$$

and

5.1.1) If $B<\frac{\left(-3 C^{4}+2(1+M) C^{3}-(M+A) C^{2}+A M\right)^{2}}{8 C^{3} L}$, the point $(C, L)$ is an attractor node.

5.1.2) If $B>\frac{\left(-3 C^{4}+2(1+M) C^{3}-(M+A) C^{2}+A M\right)^{2}}{8 C^{3} L}$, the point $(C, L)$ is an attractor focus.

5.2) a hyperbolic repellor, if and only if,

$$
-3 C^{4}+2(1+M) C^{3}-(M+A) C^{2}+A M>0
$$

and

5.1.1) If $B>\frac{\left(-3 C^{4}+2(1+M) C^{3}-(M+A) C^{2}+A M\right)^{2}}{8 C^{3} L}$, the point $(C, L)$ is a hyperbolic repellor focus surrounded by a limit cycle.

5.2.2) If $B<\frac{\left(-3 C^{4}+2(1+M) C^{3}-(M+A) C^{2}+A M\right)^{2}}{8 C^{3} L}$, the point $(C, L)$ is a hyperbolic repellor node, and the singularity $(0,0)$ is globally asymptotically stable..

The proofs are immediate from the evaluation of the Jacobian matrix and $P$. We observe that the limit cycle originated by a Hopf bifurcation increases until it coincides with the heteroclinic curve, joining the points $(M, 0)$ and $(K, 0)$, and it later disappears when this heteroclinic is broken.

The point $(C, L)$ is a repellor node when $C \rightarrow M$; then, $(0,0)$ is a global attractor; however, it is not possible for the equilibrium point $(C, L)$ to be a repellor focus and $(0,0)$ a global attractor.

\subsection{Number of limit cycles}

To determine the number of limit cycles of systems, we use the calculus of the Lyapunov quantities or Lyapunov numbers [11], which are homogeneous polynomials depending on the coefficients of the variables of system. The procedure is only shown for system (5).

Theorem 3.9. Number of limit cycles

i) In system (3) if $C=\frac{1+M}{2}$, then $\operatorname{trD} Y_{\nu}(C, L)=0$ and the equilibrium point $(C, L)$ is a weak focus of order one [11].

ii) In system (4), if $A=\frac{-3 C^{2}+2 C M+2 C-M}{2 C-M-1}<1$, then the equilibrium point $(C, L)$ is a weak focus of order one.

iii) In system (5), if $A=\frac{C^{2}\left(2 C-M+2 C M-3 C^{2}\right)}{C^{2}-M}<1$, then the equilibrium point $(C, L)$ is at least an order-two weak focus.

Proof. The procedure to establish the quantity of limit cycles is shown in system (5) with sigmoid functional response.

iii) Using the change of variables

$$
u=U+C \text { and } v=V+L
$$

we obtain the new system given by

$$
Z_{\nu}:\left\{\begin{array}{l}
\frac{d U}{d \tau}=\left(\begin{array}{c}
(1-U-C)(U+C-M)\left(A+(U+C)^{2}\right)- \\
(U+C)(V+L)
\end{array}\right)(U+C) \\
\left.\frac{d V}{d \tau}=B\left((U+C)^{2}-C^{2}\right)\right)(V+L)
\end{array}\right.
$$

The Jordan matrix associated to vector field $Z_{\nu}[3]$ is

$$
J=\left(\begin{array}{cc}
0 & -W \\
W & 0
\end{array}\right)
$$


with $W^{2}=2 C^{3} L B=\operatorname{det} D Z_{\eta}(0,0)$ and $\eta_{1}=\operatorname{tr} D Z_{\eta}(0,0)=0$. Then,

$$
A=\frac{C^{2}\left(2 C-M+2 C M-3 C^{2}\right)}{C^{2}-M}
$$

The matrix change of basis [3] is

$$
M=\left(\begin{array}{cc}
0 & -W \\
\frac{W^{2}}{C^{2}} & 0
\end{array}\right)
$$

With a long algebraic calculations and time rescaling we derive the normal form [11]

$$
\bar{Z}_{\eta}:\left\{\begin{array}{c}
\frac{d x}{d T}=-y-2 B C x y+\frac{W}{2 C} y^{2}+B W x y^{2} \\
x-\frac{2 W}{C} x y+\frac{C\left(3 C M-C^{3}+3 C^{4}+3 C M^{2}-6 C^{2} M-C^{3} M-M^{2}\right)}{C^{2}-M} y^{2} \\
\frac{d y}{d T}=\begin{array}{c}
W^{2} \\
C^{2}
\end{array} y^{2}-\frac{W\left(4 C M-2 C^{3}+7 C^{4}-M^{2}+4 C M^{2}-10 C^{2} M-2 C^{3} M\right)}{C^{2}-M} y^{3} \\
-W^{2}(M-5 C+1) y^{4}-W^{3} y^{5}
\end{array}\right.
$$

Using the Mathematica package [75], we obtain that the second Lyapunov quantity [11] is

$$
\eta_{2}=\frac{-3 C(-1+4 C-M) W}{8}
$$

and

with

$$
\eta_{3}=\frac{W}{128 C^{5}} f_{31}(M, B)
$$

$$
f_{31}(M, B)=\frac{1}{2097152} \frac{(M+1)^{8}}{M^{2}-14 M+1} f_{32}(M, B)
$$

where

$$
\begin{aligned}
f_{32}(M, B) & =6912 M\left(M^{2}-14 M+1\right) B^{2} \\
& +16(1-3 M)\left(-45+175 M-603 M^{2}-727 M^{3}+64 M^{4}\right) B \\
& +3 M\left(M^{2}-14 M+1\right)(M+1)^{2}(1-3 M)^{2}
\end{aligned}
$$

Clearly, $\eta_{2}$ changes sign when $C=\frac{1+M}{4}$. Then, the equilibrium $(C, L)$ is surrounded by at least two limit cycles. If $(C, L)$ is a two order weak focus, then $\eta_{3}$ must be the first non-zero Lyapunov quantity.

Evaluating $f_{32}(M, B)$ for different values of $M$, the following numerical situations are obtained:

$f_{32}(0, B)=16 \times(-45) B<0, f_{2}(7-4 \sqrt{3}, B)=-449.57 B<0$

and

$f_{32}(0.02, B)=99.588 B^{2}-627.87 B+4.3224 \times 10^{-2}$.

Hence, $f_{32}(0.02, B)$ is a convex parabola which intercepts with the $B$ - axis are $B=6.8843 \times 10^{-5}$ and $B=6.3046$.

Then, $f_{32}(M, B)$ changes sign and so does $\eta_{3}$, for $\left.M \in\right] 0,7-4 \sqrt{3}[$ and $B \in] 0,6.8843 \times 10^{-5}[U$ ]6. 3046, $\infty[$, respectively.

Therefore, $\eta_{4}$ must be calculated to determine the existence of a third limit cycle..

Assuming $f_{32}(M, B)<0$, it follows that the equilibrium point $(C, L)$ is surrounded by two limit cycles: the innermost unstable and the outermost stable.

The uniqueness of a stable limit cycle in system (3), established by means of the Lyapunov quantities, contradicts the results in [17], where the existence of a second limit cycle is claimed for this system.

Numerical simulations, depicted in Figure 6, show the existence of a separatrix curve and two limit cycles surrounding the equilibrium point $(C, L)$ in system $(5)$, the innermost of which is unstable from the inside, and the outermost of which is stable from the outside [48].

The results of this simulation show that the trajectories of system (5) with positive initial conditions above the separatrix curve approach the singularity $(0,0)$; those solutions with initial conditions below 


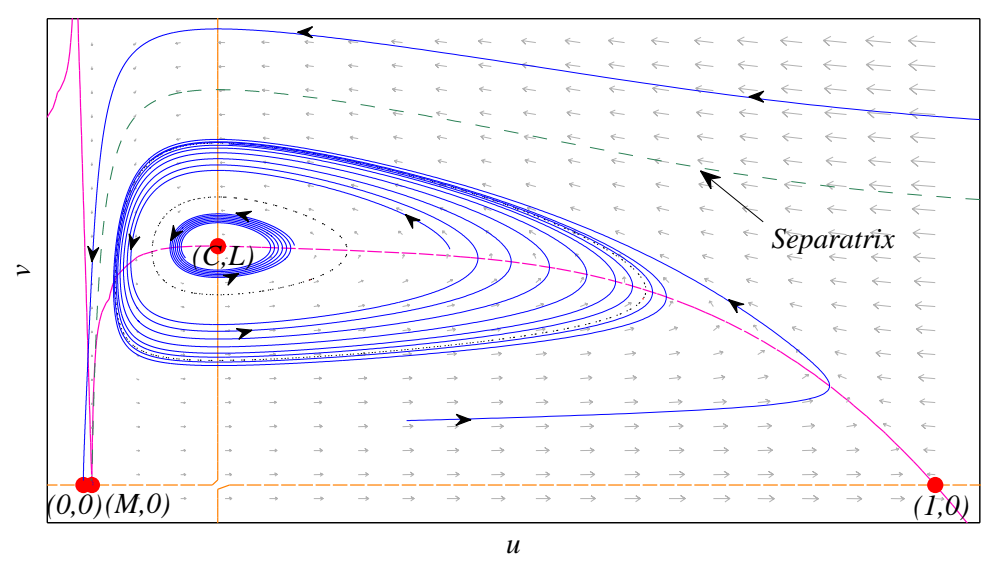

Figure 6. In system (5), for $A=0.458, B=0.1, C=0.3873$, and $M=0.01$, the equilibrium point $(C, L)=(0.3873,0.3629)$ is surrounded by two limit cycles. The separatrix curve generated by the stable manifold of $(M, 0)$ is shown by the dashed line in green.

this separatrix but outside of the unstable limit cycle tend to the stable limit cycle, and trajectories with initial conditions inside of the unstable limit cycle have the positive equilibrium point $(C, L)$ as their $\omega$-limit, demonstrating the phenomenon of metastability $[33,34,61,76]$.

\subsection{Existence of heteroclinic cycle}

Now, we will analyze the stability of the heteroclinic cycle obtained by the breaking of the heteroclinic curve determined by the stable manifold $W^{s}(M, 0)$ and the unstable manifold $W^{u}(1,0)$, of Lemma 3.4.

We apply the criterion to compute $R$, namely the modulus of the ratio between the products of the negative and positive eigenvalues of the two saddle points $P_{1}$ and $P_{2}$, that determine the hetroclinic curve (see [4] page 88). This criterion is based on the Andronov-Leontovich Theorem (see page 200 [49]) given for homoclinic bifurcations.

Denoting by $\lambda_{1}^{+}\left(P_{1}\right), \lambda_{2}^{+}\left(P_{2}\right), \lambda_{1}^{-}\left(P_{1}\right)$ and $\lambda_{1}^{-}\left(P_{2}\right)$ the eigenvalues associated to the saddle points $P_{1}$ and $P_{2}$, where the upper indices corresponds to the sign of the respective eigenvalue; then,

$$
R=\left|\frac{\lambda_{2}^{-}\left(P_{1}\right) \times \lambda_{1}^{-}\left(P_{2}\right)}{\lambda_{1}^{+}\left(P_{1}\right) \times \lambda_{2}^{+}\left(P_{2}\right)}\right| .
$$

We determine the neutrality of the heteroclinic cycle considering $R=1$; the heteroclinic cycle is stable, if $R>1$ and unstable, if $R<1$ [4].

Proposition 3.10. Stability of the heteroclinic cycle for system (3)

There exist a heteroclinic cycle, if and only if, $A>\frac{2 M}{M+1}$. This cycle is stable and coincides with the limit cycle generated by Hopf bifurcation.

Proof. Remebering the Jacobian matrix of system (3) and its evaluation in the saddle point $(M, 0)$ and $(1,0)$, we have that $R$, given by $(6)$ is

$$
R=\left|\frac{\lambda_{2}^{-}(M, 0) \times \lambda_{1}^{-}(1,0)}{\lambda_{1}^{+}(M, 0) \times \lambda_{2}^{+}(1,0)}\right|
$$




$$
\begin{aligned}
& =\left|\frac{(-B(C-M))(-(1-M))}{M(1-M) B(1-C)}\right| \\
& =\frac{|C-M|}{|M||1-C|} .
\end{aligned}
$$

The neutrality of the heteroclinic cycle is given by the condition $R=1$. Then, we obtain $C-M=$ $M(1-C)$; so $(M+1) C-2 M=0$.

Therefore, the heteroclinic cycle is neutral, if and only if, $C=\frac{2 M}{M+1}$.

In order to determine the relative positions of the stable and unstable manifolds of the saddle points $(M, 0)$ and $(1,0)$, for parameter values satisfying the obtained neutrality condition, we will compare with the condition obtained for the Hopf bifurcation.

The Hopf bifurcation is originated when $\operatorname{tr} D L_{\nu}\left(C, v_{e}\right)=0$, i.e., for $C=\frac{M+1}{2}$. [4,37].

Thus, we calculate the difference $D$ between the two curves (conditions),

$$
\begin{aligned}
& D=\frac{M+1}{2}-\frac{2 M}{M+1} \\
& =\frac{1}{2} \frac{(M-1)^{2}}{M+1}>0 .
\end{aligned}
$$

Then, the curve where the heteroclinic cycle is neutral lies below the Hopf bifurcation curve. So, the heteroclinic cycle is always stable for a wide set of values of $B$.

Proposition 3.11. Stability of the heteroclinic cycle for system (4)

The heteroclinic cycle is stable, if and only if, $A>\frac{(1-C) M^{2}+M-C}{C-M-M(1-C)}$.

The heteroclinic cycles is unstable, if and only if, $A<\frac{(1-C) M^{2}+M-C}{C-M-M(1-C)}$.

The heteroclinic cycle is neutrality stable, if and only if, $A=\frac{(1-C) M^{2}+M-C}{C-M-M(1-C)}$

Proof. Again we employ the procedure described [4] to determine the stability of the cycle heteroclinic.

The proper values are of system (4) are:

$\lambda_{1}^{+}(M, 0)=M(1-M)(M+A)>0, \lambda_{2}^{-}(M, 0)=B(M-C)<0$,

$\lambda_{1}^{-}(1,0)=-(1-M)(1+A)<0$ and $\lambda_{2}^{+}(1,0)=B(1-C)>0([34])$.

Then,

$R=\left|\frac{(M-C)(A+1)}{(1-C) M(A+M)}\right|=\frac{(C-M)(A+1)}{(1-C) M(A+M)}$.

Assuming the condition $R=1$, to obtain a constraint for the existence of the heteroclinic cycle be neutral, we have $(C-M)(A+1)=(1-C) M(A+M)$. Therefore,

$$
A=\frac{(1-C) M^{2}+M-C}{C-M-M(1-C)} .
$$

So, considering the different alternatives for condition (7), the proof is completed.

Remark 3.12. To determine the possibility of existence of a non-infinitesimal limit cycle, we calculate the difference between the curve $(B)$ with the obtained condition for the Hopf bifurcation.

We remember that

$\operatorname{tr} D H_{\eta}\left(C, v_{e}\right)=C\left((2 C-M-1) A+\left(3 C^{2}-2(M+1) C+M\right)\right)$, [34] which dependent of factor

$T=(1+M-2 C) A-\left(3 C^{2}-2(M+1) C+M\right)$.

So, $T=0$, if and only if, $A=\frac{3 C^{2}-2(M+1) C+M}{1+M-2 C}$.

The difference is

$D(M, C)=\frac{3 C^{2}-2(M+1) C+M}{1+M-2 C}-\frac{M(M+1)-\left(M^{2}+1\right) C}{(M+1) C-2 M}$

$=(1-C)(C-M) \frac{3(M+1) C-\left(M^{2}+4 M+1\right)}{(2 C-M-1)((M+1) C-2 M)}$.

The sign of $D$ dependent of

$F(M, C)=\frac{3(M+1) C-\left(M^{2}+4 M+1\right)}{(2 C-M-1)((M+1) C-2 M)}$.

Then, the sign of $F$ depends on the factors (curves) $N_{1}=3(M+1) C-\left(M^{2}+4 M+1\right), N_{2}=$ $2 C-M-1$ and $N_{3}=(M+1) C-2 M$. By a simple evaluation of them considering a fixed value for $M$ (for example $M=0.1$ ) and different values of $C$ (we choose, $C=0.1,0.2,0.45$ and 0.6 ) we can verify that $F$ can have different sign (which are $F=-13.333,53.571,-1.2712,12.391$, respectively). 
This numerical result implies that the relative position of the surfaces representing the heteroclinic bifurcation and the Hopf bifurcation can produce a new limit cycle for system (4)

Remark 3.13. The possible second limit cycle for system (4) has not been detected by simulations.

In this paper we will not analyze the existence of a heteroclinic cycle for the model (5) or in the special cases where $M=0$.

\subsection{A special case of weak Allee effect}

Now, we consider $M=0$ (i.e. $m=0$ ), as having a special case of the weak Allee effect. We have that the point $(M, 0)$ coincides with $(0,0)$, being now a nonhyperbolic equilibrium point with parabolic and hyperbolic sectors [60].

The existence of the separatrix curve is maintained, dividing the behavior of the trajectories on the phase plane as in the case of a strong Allee effect. Those orbits with initial conditions in the parabolic sector have the point $(0,0)$ as their $\omega$-limit. The orbits with initial conditions in the hyperbolic sector can have a different $\omega$-limit, according to the parameter values.

The equilibrium points of the system of differential equations (3) and (4) are:

$O=(0,0), Q_{1}=(1,0)$ and $Q_{e}=\left(C, L^{\prime}\right)$, where

$L^{\prime}=(1-C) C$, in system $(3)$ and

$L^{\prime}=(1-C)(A+C) C$, in system (4).

As previously shown, system (5), with sigmoid functional response, can have three limit cycles, but when $M=0$, this number diminishes. Now we have the system

$$
Y_{\lambda}:\left\{\begin{array}{l}
\frac{d u}{d \tau}=\left((1-u)\left(A+u^{2}\right)-v\right) u^{2} \\
\frac{d v}{d \tau}=B\left(u^{2}-C^{2}\right) v
\end{array}\right.
$$

where $\left.\lambda=(A, B, C) \in \Delta_{1}=\right] 0,1\left[\times \mathbb{R}_{+} \times \mathbb{R}_{+}\right.$. System (8) or vector field $Y_{\lambda}$ has three equilibrium points, $(0,0),(1,0)$ and $\left(C, L^{\prime}\right)$, with $L^{\prime}=(1-C)\left(A+C^{2}\right)$ and it has exactly two limit cycles.

Theorem 3.14. Number of limit cycles for $M=0$.

i) In system (3) if $C=\frac{1}{2}$, then $\operatorname{tr} D Y_{\nu}(C, L)=0$ and the equilibrium point $\left(\frac{1}{2}, L\right)$ is a weak focus of order one [11].

ii) In system (4), if $A=\frac{C(3 C-2)}{1-2 C}<1$ and $\frac{2}{3}<C<\frac{1}{2}$, then the equilibrium point $(C, L)$ is a weak focus of order one.

iii) In system (5), if $A=C(2-3 C)$, then the equilibrium point $(C, L)$ is at least a two-order weak focus.

Proof. In system (5) the new equilibrium $\left(C, L^{\prime}\right)$ is a two order weak focus since the sign of $\eta_{2}=$ $\frac{-3 C(-1+4 C) W}{8}$ changes at $C=\frac{1}{4}$ (see Theorem 3.9) and $\eta_{3}=\frac{W}{128 C^{5}} f_{1}(B)$ with $f_{1}(B)=\frac{1}{2097152} f_{2}(B)$ and $f_{2}(B)=-16 \times 45 B<0$, then $\eta_{3}<0$ (it does not change sign).

Then, when $\operatorname{tr} D Y_{\lambda}\left(C, L^{\prime}\right)<0, \eta_{2}>0$ and $\eta_{3}<0$, two limit cycles are obtained.

The uniqueness of a stable limit cycle in system (3) for $M=0$, was established calculating the Lyapunov quantities; then, the point $\left(\frac{1}{2}, L\right)$ is a first order weak focus, which contradicts an affirmation in [17], where the existence of a center is claimed.

For the knowledge of properties of system (6), the bifurcation diagram is shown in Figure 7.

It is easy to see that if $M<0$, the separatrix curve determined by the strong Allee effect disappears; then, these systems have a behavior similar to those with a compensatory growth function of the prey. The parameter $B$ determines mainly whether the positive equilibrium point is a node or focus.

The straight line $C=0.25$ in figure 7 , divides the parameter space, since the quantity $\eta_{2}$ changes sign, as is demonstrated in the proof of Theorem 3.10 . 


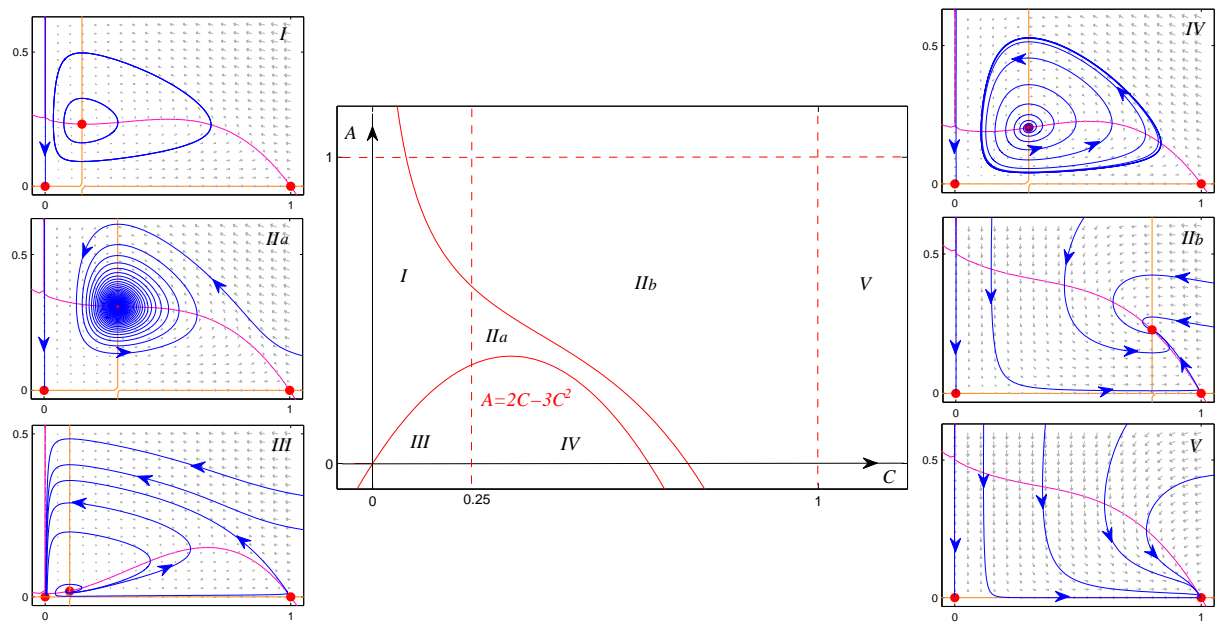

Figure 7. Bifurcation diagram of system (6), for the special case of the weak Allee effect when $M=0$ and for any fixed value of $B$. Five subsets in the $A C-$ plane of the parameter space exist, for $A<1$, in which the system exhibits different dynamics. We observe in region I of this plane, there are two limit cycles, determined by Hopf bifurcation, the outermost stable and the innermost unstable.

\section{Conclusions}

In this paper, we have concentrated on the dynamics of Gause type predator-prey models considering three standard functional responses and the most common form to express the Allee effect on prey. Principally, we investigate the consequences of the Allee effect on the number of limit cycles compared to the same model without this effect and the results are presented in terms of the rescaled parameters.

The most significant result of our analysis is that a unique positive equilibrium point of the system is either locally stable or lead to a stable limit cycle; there can also exist a range of parameters wherein multiple stable states occur.

For Gause type models with strong Allee effect and the special case $M=0$, the following common properties are obtained.

1. The point $(0,0)$ is an attractor for all parameters values.

2. There exists a separatrix curve determined by the stable manifold of the saddle point $(M, 0)$ for the strong Allee effect.

When $M=0$, the point $(M, 0)$ coincides with $(0,0)$; this point is non-hyperbolic and in this case, the separatrix curve is determined using the blowing-up method [11,20].

3. The trajectories close to the separatrix curve are highly sensitive to the initial condition. Those with initial condition below this curve, i. e., in region $\Lambda$, have as $\omega$-limit either a limit cycle or the stable positive equilibrium point. Those with initial conditions above this curve have the origin $(0,0)$ as their $\omega$-limit. Also, the sensitivity of the bifurcation structure on the formulation of the Allee effect, is shown in [63] where a ratio-dependent model is analyzed.

4. There exist parameter constraints for which the unique equilibrium point is locally asymptotically stable.

5. There are parameter conditions for which at least one limit cycle exists. This corresponds to the occurrence of a Hopf bifurcation when there is a change of stability at the unique positive equilibrium point. 
6. The diameter of this limit cycle increases until it coincides with the heteroclinic curve joining the equilibrium points $(M, 0)$ and $(1,0)$; later, this heteroclinic breaks up and the singularity $(0,0)$ is globally asymptotically stable $[33,34]$.

Some ecological interpretations of these properties are:

a) From property 1) we have that at low population sizes both species go to extinction, independent of the parameters.

b) However, extinction could occur even for initially high population sizes of the prey (near $K$ in system (2)), as seen in Figures 2, 3, 4, 5, 6 and 7.

c) From property 3), the trajectories starting near the separatrix curve are sensitive to their initial conditions, implying that small perturbations due to environmental changes caused by pollution or other causes, could provoke the extinction of both populations.

For instance, from figure 7 we can see that the conservation of both species is possible, if a quantity of predator is removed from the interaction. But, from figure 4, we can have extinction of both species if a quantity of prey is released into the environment.

d) Also, there exists the region $\Lambda$ where both species coexist, which is determined by the $u$-axis, the stable manifold $W^{s}(M, 0)$ of point $(M, 0)$ and the straight $u=1$. There, the populations coexist and tend to a stable equilibrium (from property 4) or they have an oscillatory behavior (from property 5 ).

e) From property 6) follows that, if the size of population oscillations increases, according to changes of the parameter values, the possibility of extinction of both populations also increases.

Therefore, the strong Allee effect in the prey and the special case $M=0$ for the weak Allee effect, have a great influence on the predator-prey interaction, due to the existence of the separatrix curve in the phase plane, giving rise to a large region of extinction of both populations.

On the other hand, comparing the results of theorems 6, 7 and 8, we can conclude that the inclusion of the Allee effect in a Gause type predator-prey model can have different consequences regarding the number of limit cycles compared to the respective model without this effect.

Particularly, dynamics of system (4) are more complex than the Rosenzweig-MacArthur model (without Allee effect) $[33,35]$, which has only four possible dynamics, included the case $A=1-2 C$, as shown in Figure 8.

Moreover, the Gause type predator-prey model considering the most common equation for the Allee effect in the prey with:

i) linear functional response has one limit cycle, but it is well-known that the Lotka-Volterra predatorprey model has no limit cycles $[4,22,29,53,58,70]$,

ii) hyperbolic functional response has one limit cycle as has the Rosenzweig-MacArthur predator prey model [70],

iii) sigmoid functional response has two limit cycles and the number of limit cycles increases compared to the predator prey model without Allee effect [67].

We observe that the incorporation of this ecological phenomenon into predator-prey models can increase, decrease or conserve the number of limit cycles, as shown in Table 2.

Table 2. Number of limit cycles of Gause-type models generated by Hopf bifurcation, with and without Allee effect. The superscripts indicate the references, where $[a]$ is the present work.

\begin{tabular}{|l|c|c|c|c|}
\hline Functional response $h(x) \rightarrow$ & $q x$ & $\frac{q x}{x+a}$ & $\frac{q x^{2}}{x^{2}+a}$ & $\frac{q x}{x^{2}+a}$ \\
\hline$\frac{d x}{d t}=r\left(1-\frac{x}{K}\right) x$ & $0^{[29]}$ & $1^{[37]}$ & $1^{[65]}$ & $2^{[31]}$ \\
\hline$\frac{d x}{d t}=r\left(1-\frac{x}{K}\right)(x-m) x$ & $1^{[21]}$ & $1^{[35]}$ & $3^{[a]}$ & $1^{[36]}$ \\
\hline$\frac{d x}{d t}=r\left(1-\frac{x}{K}\right) x^{2}$ & $1^{[21]}$ & $1^{[35]}$ & $2^{[a]}$ & $1^{[36]}$ \\
\hline
\end{tabular}

As shown by the analysis, the existence of limit cycles is not equivalent to the unstability of the positive critical point, in general. In figure 6, we have illustrated the existence of two limit cycles for the Gause type model with a sigmoid functional response and Allee effect on prey. The results of this 

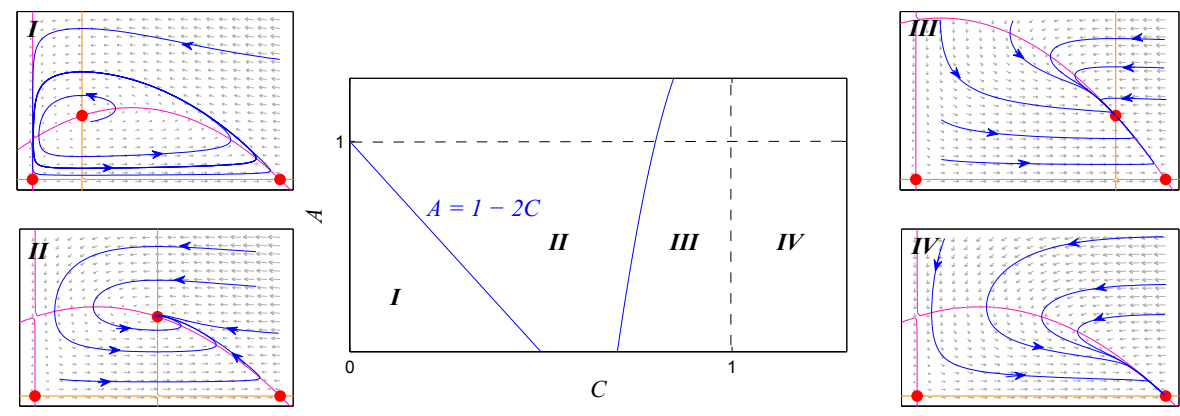

Figure 8. Bifurcation diagram of the Rosenzweig-MacArthur model, for $B$ fix. Four subsets in the $A C-$ plane of the parameter space exist, for $A<1$, in which different dynamics exist.

simulation show that the solutions of the models with positive initial conditions approach the limit cycle, or the positive equilibrium point or the singularity $(0,0)$, demonstrating the phenomenon of metastability $[33,34,61,76]$.

There are many real ecosystems that possess multiple stable states, and studying mathematical models that exhibit this behavior can lead to insights about threshold and breakpoint behavior. This means that the system is potentially capable of modeling population explosions (outbreaks) and crashes as well as stable predator-prey relationships $[16,76]$.

We also claim that the choice of the form for the functional response also determines this quantity (and the behavior of the systems). The results described above indicate that the Gause-type predator-prey models with a simple form to describe the Allee effect on prey are sensitive to a change in the way that the predator functional response is expressed mathematically [59].

We estimate that to obtain general properties for these models, in particular, the determination of conditions that guarantee the existence and uniqueness or multiplicity of limit cycles surrounding a positive equilibrium, is an interesting but challenging open problem [77].

As by changing the expression of one interaction term may have a significant effect on the stability of system and persistence of the population models [59], then in future works we will change the mathematical form to model the Allee effect (for instance, assuming the form employed in [71]), but preserving the three functional responses used here. We hope that the number of limit cycles surrounding a unique equilibrium point will undergo changes, such as those occurring in the Leslie-Gower type models $[1,33]$.

Acknowledgements. The authors thank to the anonymous referees by the helpful suggestions and comments which led to enhance the original manuscript. They also wish to thank the members of the Grupo de Ecología Matemática of the Instituto de Matemáticas at the Pontificia Universidad Católica de Valparaíso for their valuable comments and suggestions. This work is partially financed by Project Fondecyt $\mathrm{N}^{o} 1120218$ and DIEA-PUCV 124.730/2012. 


\section{References}

[1] P. Aguirre, E. González-Olivares, E. Sáez. Three limit cycles in a Leslie-Gower predator-prey model with additive Allee effect. SIAM J. Appl. Math., 69(5) (2009), 1244-1269.

[2] E. Angulo, G. W. Roemer, L. Berec, J. Gascoigne, F. Courchamp. Double Allee effects and extinction in the island fox. Conser. Biol., 21 (2007), 1082-1091.

[3] D. K. Arrowsmith, C. M. Place. Dynamical System. Differential equations, maps and chaotic behaviour, Chapman and Hall, London, 1992.

[4] A. D. Bazykin, F. S. Berezovskaya, A. S. Isaev, R. G. Khlebopros, Dynamics of forest insect density: Bifurcation approach, J. Theor. Biol., 186 (1997), 267-278.

[5] A. D. Bazykin. Nonlinear dynamics of interacting populations. Nonlinear Sciences Series A Vol. 11, World Scientific, Singapore, 1998.

[6] L. Berec, E. Angulo, F. Courchamp. Multiple Allee effects and population management. Trends Ecol. Evol., 22 (2007), 185-191.

[7] F. Berezovskaya, G. Karev, R. Arditi. Parametric analysis of the ratio-dependent predator-prey model. J. Math. Biol., 43 (2001), 221-246.

[8] D. S. Boukal, L. Berec. Single-species models and the Allee effect: Extinction boundaries, sex ratios and mate encounters. J. Theor. Biol., 218 (2002), 375-394.

[9] D. S. Boukal, M. W. Sabelis, L. Berec. How predator functional responses and Allee effects in prey affect the paradox of enrichment and population collapses. Theor. Popul. Biol., 72 (2007), 136-147.

[10] K. S. Cheng. Uniqueness of a limit cycle for a predator-prey system. SIAM J. Math. Anal., 12 (1981), $541-548$.

[11] C. Chicone, Ordinary differential equations with applications (2nd edition). Texts in Applied Mathematics 34, Springer, New York, 2006.

[12] C. W. Clark. Mathematical models in the economics of renewable resources. SIAM Rev., 21(1) (1979), 81-99.

[13] C. W. Clark, Mathematical Bioeconomics: The optimal management of renewable resources (2nd edition), John Wiley and Sons, New York, 1990.

[14] C. W. Clark. The Worldwide Crisis in Fisheries: Economic Model and Human Behavior. Cambridge University Press, Cambridge, 2007.

[15] C. S. Coleman. Hilbert's 16th problem: How many cycles?. In: M. Braun, C. S. Coleman and D. Drew (Eds.) Differential Equations Models, Springer-Verlag, New York, (1983), 279-297.

[16] J. B. Collings, D. J. Wollkind. A global analysis of a temperature-dependent model system for a mite predator-prey interaction. SIAM J. Appl. Math., 50 (1990), 1348-1372.

[17] E. D. Conway, J. A. Smoller. Global Analysis of a system of predator-prey equations. SIAM J. Appl. Math., 46 (1986), 630-642.

[18] F. Courchamp, T. Clutton-Brock, B. Grenfell. Inverse density dependence and the Allee effect. Trends Ecol. Evol., 14(10) (1999), 405-410.

[19] F. Courchamp, L. Berec, J. Gascoigne. Allee effects in Ecology and Conservation. Oxford University Press, Oxford, 2007.

[20] F. Dumortier, J. Llibre, J. C. Artés. Qualitative theory of planar differential systems. Springer, Berlin, 2006.

[21] J. D. Flores, J. Mena-Lorca, B. González-Yañez, E. González-Olivares. Consequences of depensation in a Smith's bioeconomic model for open-access fishery. In (R. Mondaini and R. Dilao Eds.) Proceedings of International Symposium on Mathematical and Computational Biology. E-papers Serviços Editoriais Ltda., Río de Janeiro, (2007), $219-232$.

[22] H. I. Freedman, Deterministic mathematical model in Population Ecology, Marcel Dekker, New York, 1980.

[23] G. F. Fussmann, B. Blasius. Community response to enrichment is highly sensitive to model structure. Biol. Lett. 1 (2004), 9-12.

[24] V. Gaiko. Global bifurcation Theory and Hilbert's sexteenth problem. Kluwer Academic Press, Boston, 2003.

[25] J. C. Gascoigne, R. Lipcius. Allee effects driven by predation. J. Appl. Ecol. 41 (2004), 801-810.

[26] G. F. Gause. The Struggle for Existence. Dover Publications Inc, London, 1971.

[27] W. M. Getz. A hypothesis regarding the abruptness of density dependence and the growth rate populations. Ecology 77 (1996), 2014-2026.

[28] L. Ginzburg, M. Colyvan. Ecological Orbits: How Planets Move and Populations Grow. Oxford University Press, Oxford, 2004.

[29] B-S. Goh, Management and Analysis of Biological Populations, Elsevier Scientific Publishing Company, Amsterdam, 1980.

[30] E. González-Olivares, R. Ramos-Jiliberto. Dynamic consequences of prey refuges in a simple model system: more prey, fewer predators and enhanced stability. Ecol. Model. 166 (2003), 135-146.

[31] E. González-Olivares, B. González-Yañez, E. Sáez, I. Szantó N. On the number of limit cycles in a predator prey model with non-monotonic functional response. Discrete Cont. Dyn-B., 6 (2006), 525-534.

[32] E. González-Olivares, B. González-Yañez, J. Mena-Lorca, R. Ramos-Jiliberto. Modelling the Allee effect: are the different mathematical forms proposed equivalents? In R. Mondaini (Ed.) Proceedings of International Symposium on Mathematical and Computational Biology. E-papers Serviços Editoriais Ltda., Río de Janeiro, (2007), 53-71.

[33] E. González-Olivares, J. Mena-Lorca, A. Rojas-Palma, J. D. Flores. Dynamical complexities in the Leslie-Gower predator-prey model considering a simple form to the Allee effect on prey. Appl. Math. Model., 3 (2011), 366-381. 
[34] E. González-Olivares, H. Meneses-Alcay, B. González-Yañez, J. Mena-Lorca, A. Rojas-Palma, R. Ramos-Jiliberto. Multiple stability and uniqueness of the limit cycle in a Gause-type predator-prey model considering the Allee effect on prey. Nonlinear Anal-Real., 12 (2011), 2931-2942.

[35] E. González-Olivares, A. Rojas-Palma. Multiple limit cycles in a Gause type predator-prey model with Holling type III functional response and Allee effect on prey. Bull. Math. Biol., 73 (2011), 1378-1397.

[36] E. González-Olivares, A. Rojas-Palma. Limit cycles in a Gause type predator-prey model with sigmoid functional response and weak Allee effect on prey. Math. Method App. Sci. 35 (2012), 963-975.

[37] E. González-Olivares, B. González-Yañez, J. Mena-Lorca, A. Rojas-Palma, J. D. Flores, Consequences of double Allee effect on the number of limit cycles in a predator-prey model, Comp. Math. App., 62 (2011), 3449-3463.

[38] B. González-Yañez, E. González-Olivares, Consequences of Allee effect on a Gause type predator-prey model with nonmonotonic functional response, In R. Mondaini (Ed.) Proceedings of the Third Brazilian Symposium on Mathematical and Computational Biology, E-Papers Serviços Editoriais Ltda, Río de Janeiro, Vol. 2 (2004), 358-373.

[39] K. Hasík. Uniqueness of limit cycle in the predator-prey system with symmetric prey isocline. Math. Biosci., 164 (2000), 203-215.

[40] K. Hasík. On a predator-prey system of Gause type, J. Math. Biol., 60 (2010), 59-74.

[41] M. Hesaaraki, S. M. Moghadas. Existence of limit cycles for predator-prey systems with a class of functional responses. Ecol. Model. 142 (2001), 1-9.

[42] F. M. Hilker, M. Langlais, H. Malchow. The Allee effect and infectious diseases: Extinction, multistability, and the (dis-)appearance of oscillations. Am. Nat., 173(1) (2009), 72-88.

[43] X-C. Huang, Y. Wang, L. Zhu. One and three limit cycles in a cubic predator-prey system. Math. Method App. Sci., 30 (2007), 501-511.

[44] T-W. Hwang. Uniqueness of limit cycles of the predator-prey system with Beddington-DeAngelis functional response. J. Math. Anal. Appl., 290 (2004), 113-122.

[45] S-B. Hsu, T-W. Hwang, Y. Kuang. Global dynamics of a predator-prey model with Hassell-Varley type functional response. Discrete Cont. Dyn. S., 10 (2008), 857-871.

[46] C. Hui, Z. Li. Distribution patterns of metapopulation determined by Allee effects. Popul. Ecol., 46 (2004), 55-63.

[47] M. Kot, Elements of Mathematical Ecology, Cambridge University Press 2001.

[48] Y. Kuang, H. I. Freedman, Uniqueness of limit cycles in Gause type models of predator-prey systems, Math. Biosci. 88 (1988) 67-84.

[49] Y. A. Kuznetsov, Elements of Applied Bifurcation Theory (2nd Edition), Springer-Verlag, New York, 1998.

[50] Y. Lamontagne, C. Coutu, C. Rousseau. Bifurcation analysis of a predator-prey system with generalised Holling type III functional response. J. Dyn. Differ. Equ., 20(3) (2008), 535-571.

[51] M. Liermann, R. Hilborn. Depensation: evidence, models and implications. Fish Fish 2 (2001), 33-58.

[52] D. Ludwig, D. D. Jones, C. S. Holling. Qualitative analysis of insect outbreak systems: the spruce budworm and forest. J. Anim. Ecol., 36 (1978), 204-221.

[53] R. M. May, Stability and complexity in model ecosystems (2nd Edition), Princeton University Press, Princeton, NJ, 2001.

[54] H. Meneses-Alcay, E. González-Olivares. Consequences of the Allee effect on Rosenzweig-MacArthur predator-prey model. In R. Mondaini (ed.) Proceedings of the Third Brazilian Symposium on Mathematical and Computational Biology BIOMAT 2003. E-papers Serviços Editoriais Ltda., Río de Janeiro, Volumen 2 (2004), 264-277.

[55] S. J. Middlemas, T. R. Barton, J. D. Armstrong, P. M. Thompson. Functional and aggregative responses of harbour seals to changes in salmonid abundance. P. Roy. Soc. B., 273 (2006), 193-198.

[56] S. M. Moghadas, B. D. Corbett. Limit cycles in a generalized Gause-type predator-prey model. Chaos Solitons Fract., 37 (2008), 1343-1355.

[57] A. Morozov, E. Arashkevich. Patterns of zooplankton functional response in communities with vertical heterogeneity: a model study. Math. Mod. Nat. Phenom., 3(3) (2008) 131-148.

[58] W. W. Murdoch, C. J. Briggs, R. M. Nisbet. Consumer-Resources Dynamics. Monographs in Population Biology 36, Princeton University Press, Princeton, 2003.

[59] M. R. Myerscough, M. J. Darwen, W. L. Hogarth. Stability, persistence and structural stability in a classical predatorprey model. Ecol. Model., 89 (1996) 31-42.

[60] L. Perko. Differential Equations and Dynamical Systems (3rd. Edition). Springer-Verlag, New York, 2001.

[61] A. Rojas-Palma, E. González-Olivares, B. González-Yañez, Metastability in a Gause type predator-prey models with sigmoid functional response and multiplicative Allee effect on prey, In R. Mondaini (Ed.) Proceedings of International Symposium on Mathematical and Computational Biology, E-papers Serviços Editoriais Ltda., Río de Janeiro, (2007), 295-321.

[62] G. Seo and M. Kot. A comparison of two predator-prey models with Holling's type I functional response. Math. Biosci., 212 (2008) 161-179.

[63] M. Sen, M. Banerjee, A, Morozov. Bifurcation analysis of a ratio-dependent prey-predator model with the Allee effect. Ecol. Complex., 11 (2013), 12-27.

[64] P. D. Spencer, J. S. Collie. A simple predator-prey model of exploited marine fish populations incorporating alternative prey. ICES J. Mar. Sci., 53 (1995), 615-628.

[65] P. A. Stephens, W. J. Sutherland. Consequences of the Allee effect for behaviour, ecology and conservation. Trends Ecol. Evol., 14 (1999), 401-405. 
[66] P. A. Stephens, W. J. Sutherland, R. P. Freckleton. What is the Allee effect?. Oikos 87 (1999), 185-190.

[67] J. Sugie, K. Miyamoto, K. Morino. Absence of limits cycle of a predator-prey system with a sigmoid functional response. Appl. Math. Lett., 9 (1996), 85-90.

[68] J. Sugie, R. Kohno, R. Miyazaki, On a predator-prey system of Holling type. P. Am. Math. Soc., 125 (1997), $2041-2050$.

[69] R. J. Taylor. Predation. Chapman and Hall, New York,1984.

[70] P. Turchin. Complex population dynamics. A theoretical/empirical synthesis. Mongraphs in Population Biology 35 Princeton University Press, Princeton, NJ, 2003.

[71] G. A. K. van Voorn, L. Hemerik, M. P. Boer, B. W. Kooi. Heteroclinic orbits indicate overexploitation in predator-prey systems with a strong Allee effect. Math. Biosci., 209 (2007), 451-469.

[72] K. Vilches-Ponce, E. González-Olivares. A Gause-type predator-prey model with a non-differentiable functional response. (2013) submitted.

[73] M-H. Wang, M. Kot, Speeds of invasion in a model with strong or weak Allee effects, Math. Bioci., 171 (2001), 83-97.

[74] J. Wang, J. Shi, J. Wei. Predator-prey system with strong Allee effect in prey. J. Math. Biol., 62 (2011), $291-331$.

[75] S. Wolfram Research, Mathematica: A System for Doing Mathematics by Computer (2nd edition), Wolfram Research, Addison Wesley 1991.

[76] D. J. Wollkind, J. B. Collings, J. A. Logan. Metastability in a temperature-dependent model system for predator-prey mite outbreak interactions on fruit trees. Bull. Math. Biol., 50(4) (1988), 379-109.

[77] D. Xiao, Z. Zhang. On the uniqueness and nonexistence of limit cycles for predator-prey systems. Nonlinearity 16 (2003), 1185-1201.

[78] J. Zu, M. Mimura. The impact of Allee effect on a predator-prey system with Holling type II functional response. Appl. Math. Comp., 217 (2010), 3542-3556. 